\title{
Solar Meridional Flow in the Shallow Interior during the Rising Phase of Cycle 24
}

\author{
Junwei Zhao $^{1}$, A. G. Kosovichev ${ }^{2}$, and R. S. Bogart ${ }^{1}$
}

\begin{abstract}
Solar subsurface zonal and meridional-flow profiles during the rising phase of solar cycle 24 are studied using time-distance helioseismology technique. The faster zonal bands in the torsional-oscillation pattern show strong hemispheric asymmetries and temporal variations in both width and speed. The faster band in the northern hemisphere is located closer to the equator than the band in the southern hemisphere, and migrates past the equator when the magnetic activity in the southern hemisphere is reaching maximum. The meridional-flow speed decreases substantially with the increase of magnetic activity, and the flow profile shows two zonal structures in each hemisphere. The residual meridional flow, after subtracting a mean meridional-flow profile, converges toward the activity belts and shows faster and slower bands like the torsional-oscillation pattern. More interestingly, the meridional-flow speed above latitude $30^{\circ}$ shows an anti-correlation with the poleward-transporting magnetic flux, slower when the following-polarity flux is transported and faster when the leadingpolarity flux is transported. It is expected that this phenomenon slows the process of magnetic cancellation and polarity reversal in the high-latitude areas.
\end{abstract}

Subject headings: Sun: helioseismology — Sun: oscillations — Sun: interior — Sun: rotation

\section{Introduction}

Solar interior rotation and meridional circulation are global-scale flows closely related to solar dynamo and solar cycles (Charbonneau 2010), and have been widely studied using different helioseismic methods. Temporal variations of the rotation and meridional flow were a focus of study by many authors (e.g., Howe et al. 2000; Vorontsov et al. 2002; Chou \& Dai 2001; Basu \& Antia 2003; Haber et al. 2002; Zhao \& Kosovichev 2004; Komm et al. 2005; González Hernández et al.

\footnotetext{
${ }^{1}$ W. W. Hansen Experimental Physics Laboratory, Stanford University, Stanford, CA 94305-4085

${ }^{2}$ Big Bear Solar Observatory, New Jersey Institute of Technology, Big Bear City, CA 92314-9672
} 
2008; Komm et al. 2013). The global rotation exhibits faster and slower rotational bands, known as torsional oscillation, with one lower branch migrating toward the equator together with the activity belts and one higher branch drifting poleward in each hemisphere (Howe et al. 2000, 2009). For the temporal variation of the meridional flow, it was reported that the residual meridional flow, obtained after removal of a quiet-period profile, converged toward the solar activity belts in the shallow interior (e.g., Zhao \& Kosovichev 2004; Gizon 2004), but diverged from the activity belts in the deeper regions (e.g., Chou \& Dai 2001; Beck et al. 2002). Later, González Hernández et al. (2010) reported that the shallow converging pattern not just existed during the active period but also extended to the solar minimum years. Using non-helioseismic method by tracking motions of magnetic elements, Hathaway \& Rightmire (2011) reported a similar converging pattern on the solar surface. It was suggested that this phenomenon was related to the increased radiative loss in the activity belts (Gizon \& Rempel 2008). Moreover, it was found that the meridional flow speed was faster when the Sun was quiet and became slower when activities grew stronger (e.g., Basu \& Antia 2003; Zaatri et al. 2006). Hathaway \& Rightmire (2010) also reported that the meridional-flow speed at the surface substantially increased after the activity maximum of cycle 23 , which they suggested might have caused the unusually long activity minimum after that cycle.

Solar cycle 24 so far shows unusually low activities and also exhibits some unusual signs for the next cycle. Through comparing torsional oscillations of cycles 23 and 24, Howe et al. (2013) found that the internal solar rotation in higher latitude slowed down in cycle 24 from its previous cycle. Using coronal Fe XIV line observations, Altrock (2013) found that cycle 24 differed from previous cycles with no polar-crown prominences rushing to the poles. Meanwhile, observation of sunspots showed that the magnetic field strength of the sunspots was steadily decreasing since the beginning of the previous cycle (Livingston et al. 2012). All these observations have demonstrated that cycle 24 is an unusual cycle, and the next cycle seems to be unusual, too. Therefore, it is important to study the temporal evolution of the interior dynamics of the Sun in the hope that these properties can shed light on understanding this special solar cycle.

In this Letter, using the first 3.7 years data acquired by SDO/HMI during the rising phase of cycle 24, we study the torsional oscillation and meridional-flow patterns from the surface to about $20 \mathrm{Mm}$ in depth. In addition to confirming some previously reported results, we find some new properties of solar rotation and meridional flow. The faster band in the northern hemisphere migrates past the equator into the southern hemisphere by the time magnetic activities reach maximum in the southern hemisphere. The meridional-flow speed above latitude $30^{\circ}$ shows an anti-correlation with the poleward-transporting magnetic flux, slower (faster) when the followingpolarity (leading-polarity) flux is transported toward the pole. It is unclear whether these newly detected properties are common in most solar cycles or are particular just for this special cycle, but understanding the dynamic implications of these properties is crucial to understand this solar cycle with particularly low activity level, as well as the general problems like the magnetic polarity 
reversal and solar dynamo. We describe our data analysis procedure in $\S 2$, present results in $\S 3$, and discuss possible artifacts and implications of our results in $\S 4$.

\section{Data Analysis}

The Helioseismic and Magnetic Imager (HMI; Scherrer et al. 2012; Schou et al. 2012) onboard the Solar Dynamics Observatory (SDO; Pesnell et al. 2012) provides continuous observations of the full-disk photosphere. A time-distance data-analysis pipeline was developed and routine computations are carried out every day at the Joint Science Operations Center (JSOC) for SDO (Zhao et al. 2012a). The pipeline provides subsurface flow field and acoustic wave-speed perturbation maps up to a depth of $20 \mathrm{Mm}$ every 8 hours, covering most of the solar disk with an area of $120^{\circ} \times 120^{\circ}$. For details of the pipeline procedure, please see Zhao et al. (2012a). The analysis presented in this Letter, using the subsurface flow velocities directly from the time-distance pipeline, covers 2010 May 1 through 2014 January 14, a total of 3.7 years during the rising phase of solar cycle 24. This period has a total of 50 Carrington rotations, from CR 2096 (this rotation has $70 \%$ coverage, and all other rotations have full coverage) through CR 2145.

For each Carrington rotation, we average subsurface flow fields for the east-west and northsouth flow speeds at different depths. The average is performed along a $30^{\circ}$-wide central meridian band so that the systematic center-to-limb effect in the meridional-flow measurements can be readily removed following the prescription by Zhao et al. (2012b). Once the north-south flow as a function of latitude is obtained, a few corrections are needed before these flows can truly represent meridional flow. First, the systematic center-to-limb effect is removed. The center-to-limb effect remains approximately unchanged through the analysis period, however, the correction should take into account of the annual B-angle variation. Second, the effect caused by $p$-angle, the angle between the solar rotational axis with the camera axis, must be removed. The Venus transit on 2012 June 5 helped the HMI team to determine a $p$-angle of 0.07 (Jesper Schou, private communication), and the time-distance pipeline incorporated this correction starting on 2012 August 1. Results before that date were retrospectively corrected. The third systematic effect comes from the error in the Carrington elements, which also shows annual changes (Beck \& Giles 2005), and this effect is removed by subtracting a fit to an annual sinusoidal variation of the cross-equator flow speed. Subsurface meridional-flow profiles are obtained after all the above-mentioned effects are removed from the north-south flows. The annual B-angle variation also causes some effects, though small, in determining the rotation speed, and an annual sine fit is subtracted as well to obtain the subsurface rotation speed from the averaged east-west flow speed.

To better understand the connection of subsurface flow properties with solar magnetic activity, the line-of-sight magnetic field from HMI is also averaged for each Carrington rotation to 
compare with the helioseismic results. Figure 1 shows the longitudinally averaged net magnetic field strength and unsigned magnetic field strength. It is well known that for each 11-yr solar cycle, one hemisphere often has a leading magnetic polarity and a following polarity with opposite sign. The polarity switches sign in the cycle that follows. As shown in Figure 1a, for cycle 24 the leading polarity is negative (positive) in the northern (southern) hemisphere, and in each hemisphere more following-polarity magnetic flux is transported poleward than leading-polarity flux. Figure $1 \mathrm{~b}$ shows that the northern hemisphere had its maximum activity during September through December 2011, and the activity in the southern hemisphere was reaching its maximum during October 2013 through January 2014 (still ongoing when this analysis was done), nearly 2 years behind the maximum of the northern hemisphere.

\section{Results}

\subsection{Torsional Oscillation}

Torsional oscillation exhibits itself as zonal bands of faster and slower rotation, with the lowerlatitude branches migrating toward the equator together with the activity belts, and the higherlatitude branches migrating toward the poles. Torsional oscillation is often obtained by subtracting a mean rotation profile from the rotation profile of each Carrington rotation. The mean profile is ideally obtained from a period of one solar cycle, and different mean profiles may result in different pictures of torsional oscillation (Howe et al. 2013). In this study, as we only have 3.7 years data available, the mean rotation profile is averaged from the first 3 years, similar to what Howe et al. (2013) did in their analysis. This may result in an imperfect determination of the torsional oscillation, but we limit our study to only the temporal variation.

As shown in Figure 2, the faster rotation band of the equatorward-migrating branch is on the equatorial side of the activity belts and the slower band on the poleward side. The torsional oscillation exhibits little change with depth, up to $21 \mathrm{Mm}$, consistent with results from the globalhelioseismology analyses (e.g., Howe et al. 2013). Our results also show that both the width and strength of the lower-latitude branch of the torsional oscillation change with time. The faster bands became weak and narrow after the activity maximum of the northern hemisphere, i.e., close to the end of the year 2011. The temporal variation of the torsional-oscillation profile is qualitatively consistent with global helioseismology results using HMI data but not GONG data (cf. both panels in Figure 9 of Komm et al. 2014).

Our torsional oscillation results also exhibits a hemispheric asymmetry. Throughout the analysis period, the faster band in the equatorward-migrating torsional-oscillation branch is on average approximately $3^{\circ}$ closer to the equator in the northern hemisphere than in the southern hemisphere. 
Moreover, the faster band in the northern hemisphere extended past the equator into the southern hemisphere during the time the southern hemisphere was reaching its activity maximum. However, it is noteworthy that the recent ring-diagram analysis results did not seem to show this phenomenon (Komm et al. 2014).

\subsection{Decrease in Meridional-Flow Speed}

The meridional-flow profiles at selected depths averaged over one-year span for three consecutive years is shown in Figure 3, and the profiles also exhibit a hemispheric asymmetry. The largest flow speed in the northern hemisphere, which showed strong magnetic activities 2 yrs earlier than the southern hemisphere, is between $7^{\circ}$ and $13^{\circ}$, while the largest flow speed in the southern hemisphere is between $13^{\circ}$ and $18^{\circ}$, roughly $5^{\circ}$ farther away from the equator. With the increase of magnetic activity, meridional-flow speed decreases substantially at all depths above the latitude $20^{\circ}$ in both hemispheres. From June 2010 through May 2013, the flow speed at the depth of 0 $1 \mathrm{Mm}$ drops an average of $6.3 \pm 1.1 \mathrm{~m} \mathrm{~s}^{-1}$ between $13^{\circ}$ and $30^{\circ}$ in the northern hemisphere, but only drops $3.6 \pm 0.7 \mathrm{~m} \mathrm{~s}^{-1}$ between $18^{\circ}$ and $35^{\circ}$ in the southern hemisphere. At the deeper depth of $7-10 \mathrm{Mm}$, the flow speed decreases even more than at the shallower depths. At some latitudes, $25^{\circ}-40^{\circ} \mathrm{N}$ and $30^{\circ}-45^{\circ} \mathrm{S}$, the meridional flow even reverses directions, but the amount of reversal is similar to the size of error bars.

The meridional-flow profile shows two zonal structures in each hemisphere, and the size of these zonal structures changes with the evolution of the solar cycle. This is particularly clear in the deeper depth shown in Figure $3 b$. Select the green curve in Figure $3 b$ as an example: there is one zone between latitudes $0^{\circ}$ and flow velocity minimum at about $27^{\circ} \mathrm{N}$, and another zone above $27^{\circ} \mathrm{N}$. The lower latitude zone becomes smaller while the magnetic activity belts migrate toward the equator. The zonal structures look similar to what Schad et al. (2013) recently reported, but their results were for a larger depth.

\subsection{Temporal Variation of Meridional Flow}

Following the same procedure as for obtaining the torsional oscillation (Figure 2), we subtract mean meridional-flow profiles, averaged from the first 3 years, from all the meridional-flow profiles of each Carrington rotation and get the residual meridional-flow profiles. Figure 4 displays these residual flow profiles as functions of latitude and depth, with the residual flow in the southern hemisphere plotted with a reversed sign for a better visualization.

Similar to what was previously reported (e.g., Zhao \& Kosovichev 2004; González Hernández et al. 
2010), the residual meridional flow converges toward the activity belts in both hemispheres. The residual flows also display faster (or poleward) and slower (or equatorward) bands migrating toward the equator, just like the torsional oscillation, up to a depth of $13 \mathrm{Mm}$. Between 13 and $21 \mathrm{Mm}$ our result does not show an organized pattern. Compared with the torsional oscillation (Figure 2), the residual meridional-flow bands are more fragmented and wider, with the maximum speed $\sim 2 \mathrm{~m} \mathrm{~s}^{-1}$ faster than the maximum torsional-oscillation speed.

A comparison between Figure 1 $1 \mathrm{a}$ and Figure $4 \mathrm{a}$ indicates that an anti-correlation exists between the residual-flow speed and the net magnetic field strength above the latitude $\sim 30^{\circ}$ in both hemispheres. For example, in the northern hemisphere, between CR 2110 and CR 2120, there is a branch of equatorward residual flow, and at about the same time there is a poleward transport of the following-polarity (positive) magnetic flux. A similar example can be found in the southern hemisphere between CR 2120 and CR 2130. These above-mentioned areas also show some enhancement in the unsigned magnetic field strength (Figure 1b), but the correlation between the residual-flow speed and the unsigned magnetic field, generally below 0.4 depending on latitude, is not as high as the correlation between the flow speed and the net magnetic field, up to 0.86 at the latitudes displayed in Figure 5 ,

To better illustrate this anti-correlation between the meridional flow and the net magnetic field, we average both quantities along the latitudinal bands of $35^{\circ}-40^{\circ}$ in the northern hemisphere and $40^{\circ}-45^{\circ}$ in the southern hemisphere (see Figure 5). The general trend for the meridional flow is that the speed decreases with the rise of magnetic activity, as already presented in $\$ 3.2$; however, superimposing on this general trend is a component of that the flow speed is anti-correlated with the net magnetic field. That is, the poleward flow speed is faster when the leading-polarity field is transported toward the pole and slower when the following-polarity flux is transported. It is true that these two quantities show better anti-correlation in the selected latitudinal bands than in the other bands above latitude $30^{\circ}$, as the flow speed and the net magnetic flux become out of phase above the selected latitudinal bands (compare Figures $1 \mathrm{a}$ and $4 \mathrm{a}$ ).

\section{Discussion}

Through analyzing the subsurface flow fields obtained from the HMI time-distance dataanalysis pipeline, we have studied the zonal-flow and meridional-flow profiles during the rising phase of solar cycle 24. The torsional oscillation from our analysis shows strong temporal variation and hemispheric asymmetry, and the faster band in the northern hemisphere is often closer to the equator than its southern counterpart, even extending past the equator near the end of the analysis period. Meridional-flow speed above the latitude of $20^{\circ}$ drops substantially with the rise of magnetic activity, and exhibits two zonal structures below latitude $55^{\circ}$ in each hemisphere. 
From the surface to a depth of $13 \mathrm{Mm}$, the residual meridional flow, after a mean flow profile is removed, converges toward and migrates together with the activity belts. More interestingly, the meridional-flow speed above latitude $30^{\circ}$ shows an anti-correlation with the net magnetic field, i.e., the poleward flow slows down (speeds up) when the following-polarity (leading-polarity) magnetic flux is transported poleward.

We believe the results reported above are unlikely artifacts caused by surface magnetic field due to the following reasons. Our results of torsional oscillation are qualitatively consistent with the results from global helioseismology, a method believed not contaminated by the surface magnetic field (Howe et al. 1999). The converging residual meridional flow toward the activity belts is consistent with the non-helioseismic method of tracking motions of magnetic elements Hathaway \& Rightmire 2011). Ring-diagram analysis presented evidence of that the converging flow trend extended beyond the active period into the quiet period (González Hernández et al. 2010), demonstrating that these properties were not due to the surface magnetism. In this study, the meridional-flow speed above $30^{\circ}$, where no active regions appear, shows a substantial anti-correlation with the net magnetic field, but shows little correlation with the total unsigned magnetic field. Moreover, our experiments with and without masking the regions, where magnetic field strength is greater than $100 \mathrm{Gs}$, show that these magnetized regions do not cause a change of final results larger than the measurement uncertainties, mostly because the total area of these regions is just a small fraction of the Sun. All these evidences support that the above-reported results are truly solar phenomena instead of magnetic artifacts.

As shown in Figure 5, the following-polarity magnetic flux, which is transported to the polar region and expected to cause the magnetic polarity reversal, is often associated with slower meridional-flow speed, while the leading-polarity flux, which is transported poleward to strengthen the high-latitude magnetic field, is often associated with faster flow speed. Since the helioseismically measured meridional-flow speed is similar to the flux transport speed (Švanda et al. 2007), it is expected that this phenomenon slows down the magnetic cancellation in the high-latitude areas, hence delays the polarity reversal and the start of next solar cycle, according to the fluxtransport dynamo theory (Choudhuri et al. 1995; Dikpati \& Charbonneau 1999). It is possible that the observed phenomenon of the poleward meridional-flow slow-down is more closely associated in time with the relatively strong magnetic activities in the lower latitude than associated with the poleward-transporting magnetic flux; however, the effective outcome is that the following-polarity magnetic flux is transported toward the pole with a lower speed than the leading-polarity magnetic flux. This is an interesting phenomenon worth further monitoring to see whether it continues in the late phase of cycle 24 and in other solar cycles. The physical cause of this phenomenon, how it affects the solar dynamo, and what it implies for the activity level of the next solar cycle are intriguing questions requiring more observational and modeling efforts. 
We believe it is essential to examine past solar cycles to see whether the phenomena reported in this Letter are common in most solar cycles or particular for this cycle with unusually low activities. SOHO/MDI (Solar and Heliospheric Observatory / Michelson Doppler Imager; Scherrer et al. 1995) only had about two months continuous coverage each year during 1996 through 2010, making it difficult to examine these phenomena. GONG (Global Oscillation Network Group), which began observations suitable for local-helioseismology analysis in 2002, seems to provide a unique opportunity to examine the previous solar cycle.

We thank the anonymous referee for thoroughly and carefully reading our manuscript, as well as giving numerous constructive comments that help to improve the quality of this paper. SDO is a NASA mission, and HMI project is supported by NASA contract NAS5-02139 to Stanford University.

\section{REFERENCES}

Altrock, R. C. 2014, Sol. Phys., 289, 623

Basu, S., \& Antia, H. M. 2003, ApJ, 585, 553

Beck, J. G., \& Giles, P. 2005, ApJ, 621, L153

Beck, J. G., Gizon, L., \& Duvall, T. L., Jr. 2002, ApJ, 575, L47

Charbonneau, P. 2010, Living Rev. Sol. Phys. 7, 3

Chou, D.-Y., \& Dai, D.-C. 2001, ApJ, 559, L175

Choudhuri, A. R., Schüssler, M., Dikpati, M. 1995, A\&A, 303, L29

Dikpati, M., \& Charbonneau, P. A. 1999, ApJ, 518, 508

Gizon, L. 2004, Sol. Phys., 224, 217

Gizon, L., \& Rempel, M. 2008, Sol. Phys., 251, 241

González Hernández, I., Howe, R., Komm, R., \& Hill, F. 2010, ApJ, 713, L16

González Hernández, I., Kholikov, S., Hill, F., Howe, R., \& Komm, R. 2008, Sol. Phys., 252, 235

Haber, D. A., Hindman, B. W., Toomre, J., Bogart, R. S., Larsen, R. M., \& Hill, F. 2002, ApJ, 570, 855 
Hathaway, D. H., \& Rightmire, L. 2010, Science, 327, 1350

Hathaway, D. H., \& Rightmire, L. 2011, ApJ, 729, 80

Howe, R., Christensen-Dalsgaard, J., Hill, F., Komm, R., Larsen, R. W., Schou, J., Thompson, M. J., \& Toomre, J. 2000, ApJ, 533, L163

Howe, R., Christensen-Dalsgaard, J., Hill, F., Komm, R., Larson, T. P., Rempel, M., Schou, J., \& Thompson, M. J. 2013, ApJ, 767, L20

Howe, R., Christensen-Dalsgaard, J., Hill, F., Komm, R., Schou, J., \& Thompson, M. J. 2009, ApJ, 701, L87

Howe, R., Komm, R., \& Hill, F. 1999, ApJ, 524, 1084

Komm, R. González Hernández, I., Hill, F., Bogart, R., Rabello-Soares, M. C., \& Haber, D. 2013, Sol. Phys., 287, 85

Komm, R., Howe, R., González Hernández, I., \& Hill, F. 2014, Sol. Phys., in press. DOI: 10.1007/s11207-014-0490-1

Komm, R., Howe, R., Hill, F., González Hernández, I., Toner, C., \& Corbard, T. 2005, ApJ, 631, 636

Livingston, W., Penn, M. J., \& Svalgaard, L. 2012, ApJ, 757, L8

Pesnell, W. D., Thompson, B. J., \& Chamberlin, P. C. 2012, Sol. Phys., 275, 3

Schad, A., Timmer, J., \& Roth, M. 2013, ApJ, 778, L38

Scherrer, P. H., et al. 1995, Sol. Phys., 162, 129

Scherrer, P. H., et al. 2012, Sol. Phys., 275, 207

Schou, J., et al. 2012, Sol. Phys., 275, 229

Švanda, M., Kosovichev, A. G., \& Zhao, J. 2007, ApJ, 670, L69

Vorontsov, S. V., Christensen-Dalsgaard, J., Schou, J., Strakhov, V. N., \& Thompson, M. J. 2002, Science, 296, 101

Zaatri, A., Komm, R., González Hernández, I., Howe, R., \& Corbard, T. 2006, Sol. Phys., 236, 227 


$$
-10-
$$

Zhao, J., Couvidat, S., Bogart, R. S., Parchevsky, K. V., Birch, A. C., Duvall, T. L., Jr., Beck, J. G., Kosovichev, A. G., \& Scherrer, P. H. 2012a, Sol. Phys., 275, 375

Zhao, J., \& Kosovichev, A. G. 2004, ApJ, 603, 776

Zhao, J., Nagashima, K., Bogart, R. S., Kosovichev, A. G., \& Duvall, T. L., Jr. 2012b, ApJ, 749, L5 

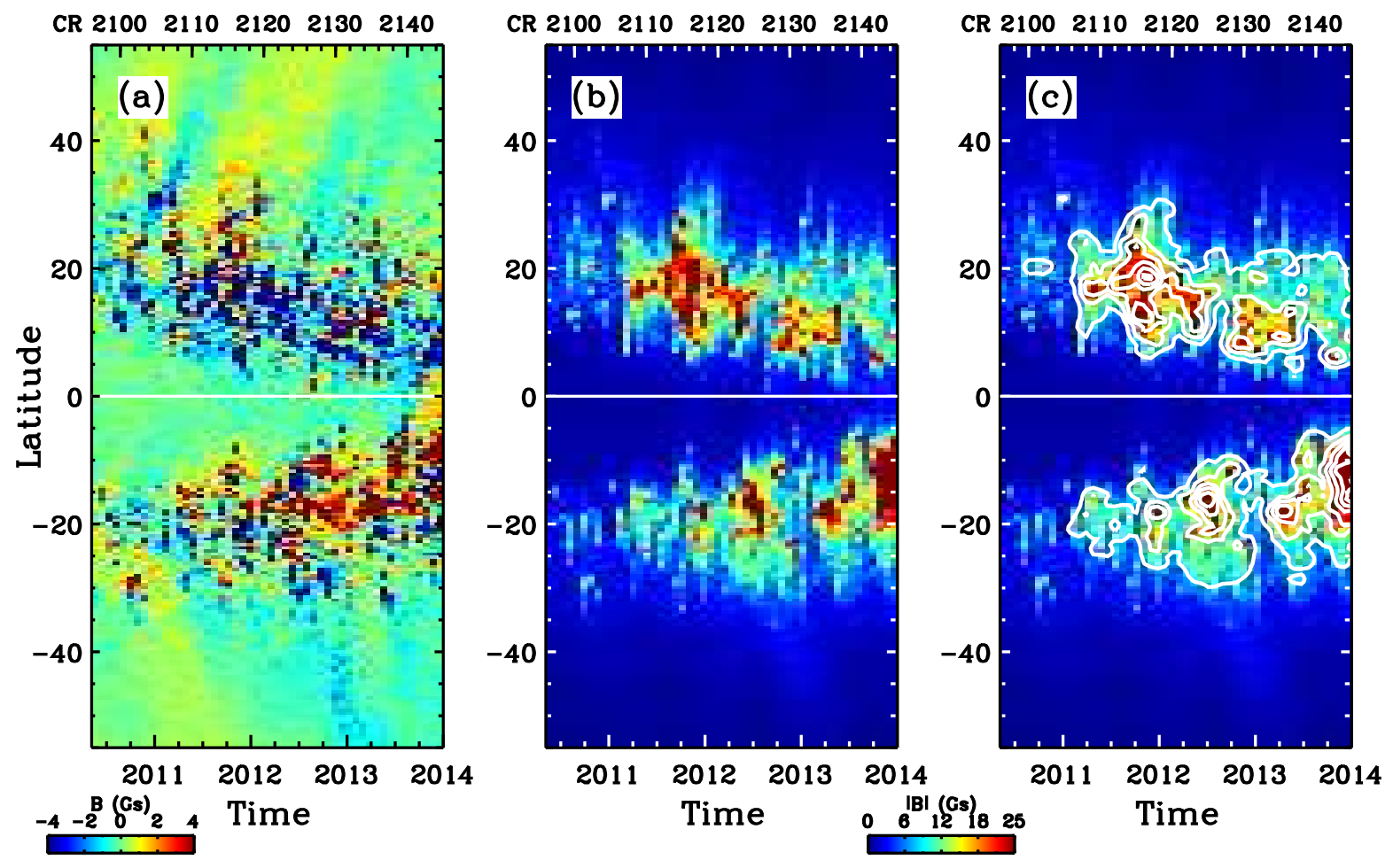

Fig. 1.- (a) Butterfly diagram of net magnetic field, obtained by averaging over all longitudes for each Carrington rotation, for the analysis period. (b) Same as panel (a) but for unsigned magnetic field. (c) Background image is the same as in (b), and contours show levels of 10, 15, 20, 25, and 30 Gauss. For all panels here and in Figures 2 and 4 , the lower horizontal axis labels years and the upper horizontal axis labels Carrington rotation numbers. 

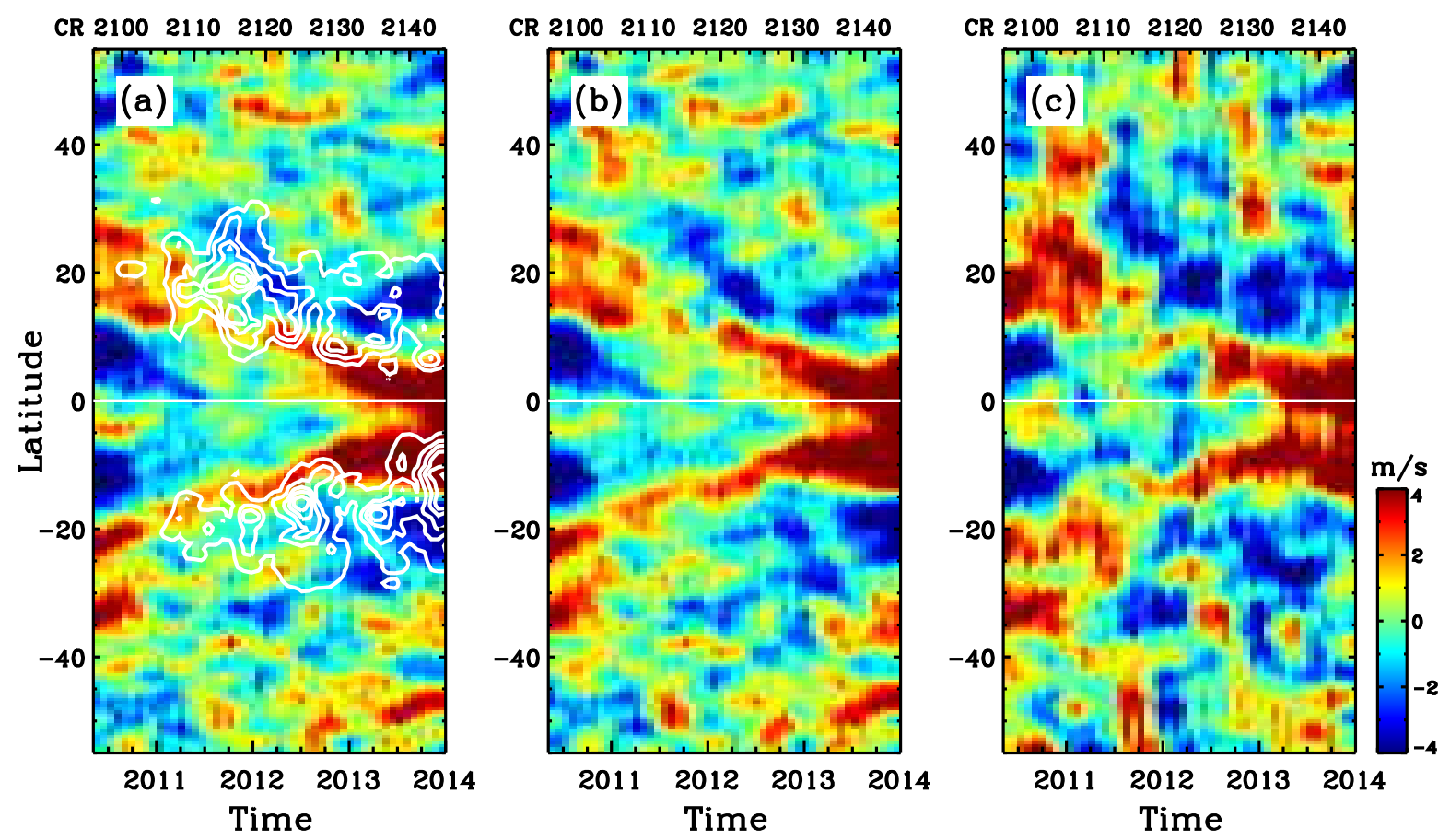

Fig. 2.- Torsional oscillation showing zonal flow after subtracting the mean rotational flow profile, for the depths of (a) $0-1 \mathrm{Mm}$, (b) $3-5 \mathrm{Mm}$, and (c) $17-21 \mathrm{Mm}$. White contours in panel (a) are the same as those in Figure 1k, indicating the location of activity belts.
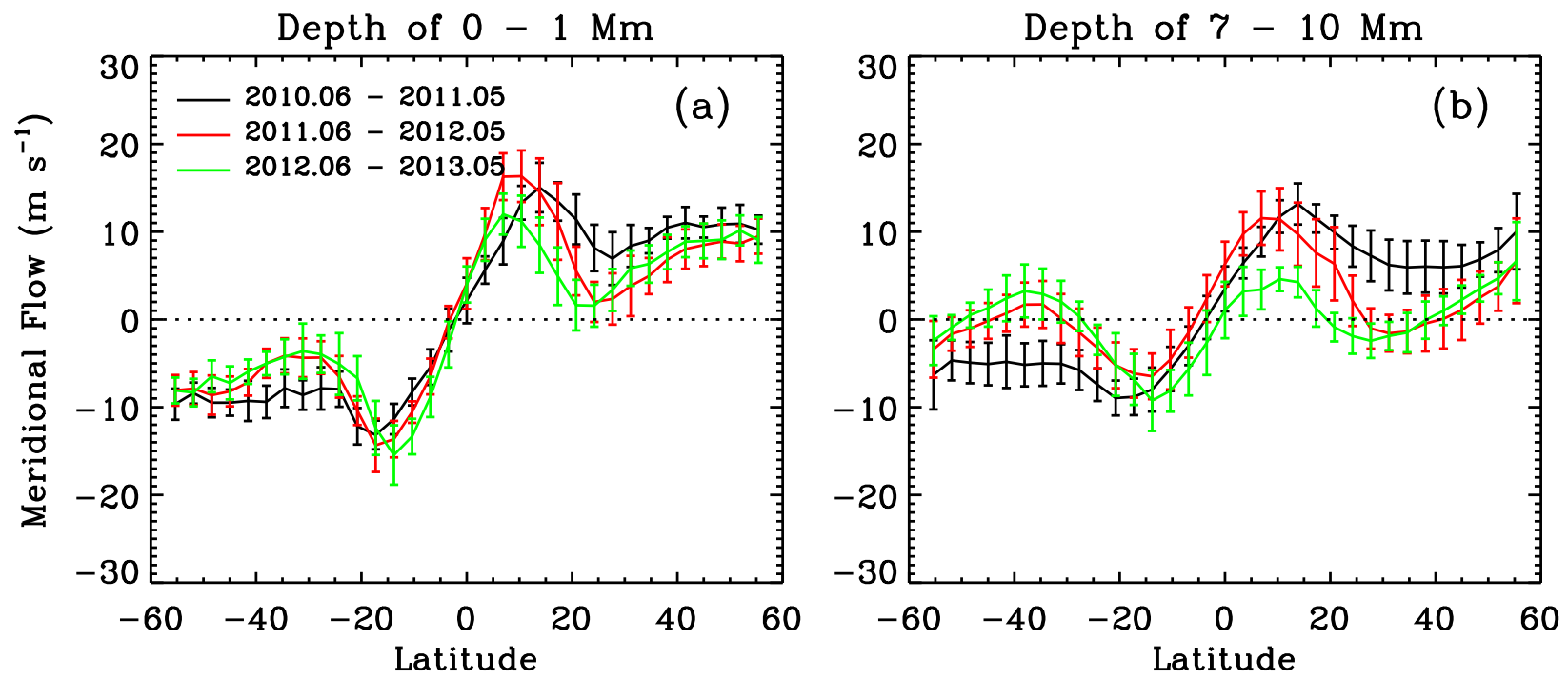

Fig. 3.- Mean meridional-flow profiles averaged from three consecutive years (represented by different colors) for two selected depths (a) $0-1 \mathrm{Mm}$ and (b) $7-10 \mathrm{Mm}$. 

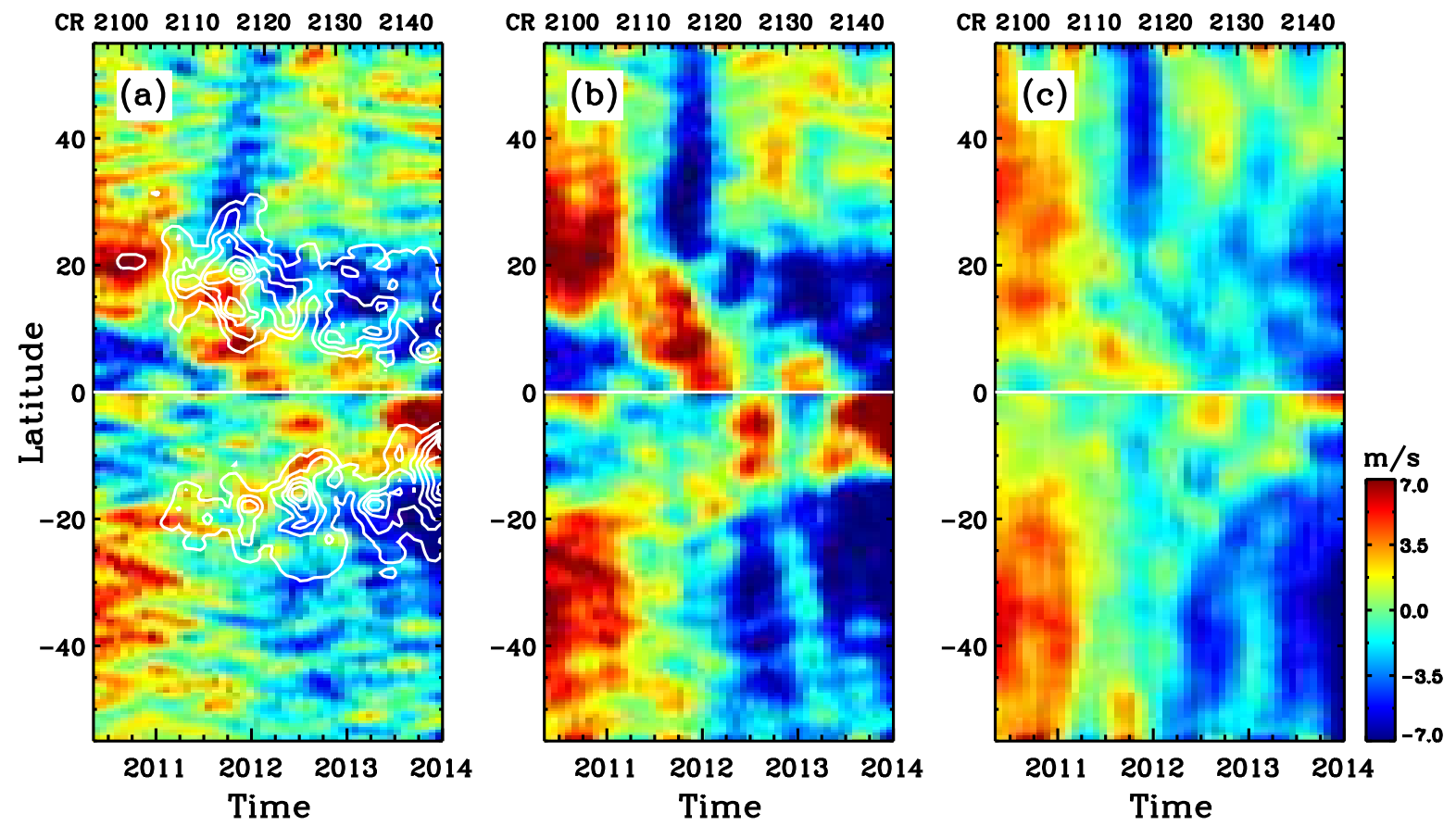

Fig. 4.- Residual meridional flow after subtraction of the mean meridional-flow profiles, for the depths of (a) $0-1 \mathrm{Mm}$, (b) $3-5 \mathrm{Mm}$, and (c) $10-13 \mathrm{Mm}$. The flow in the southern hemisphere is plotted with a reversed sign for a better visualization. Positive flow is poleward and negative flow is equatorward. White contours in panel (a) are the same as those in Figure 16.
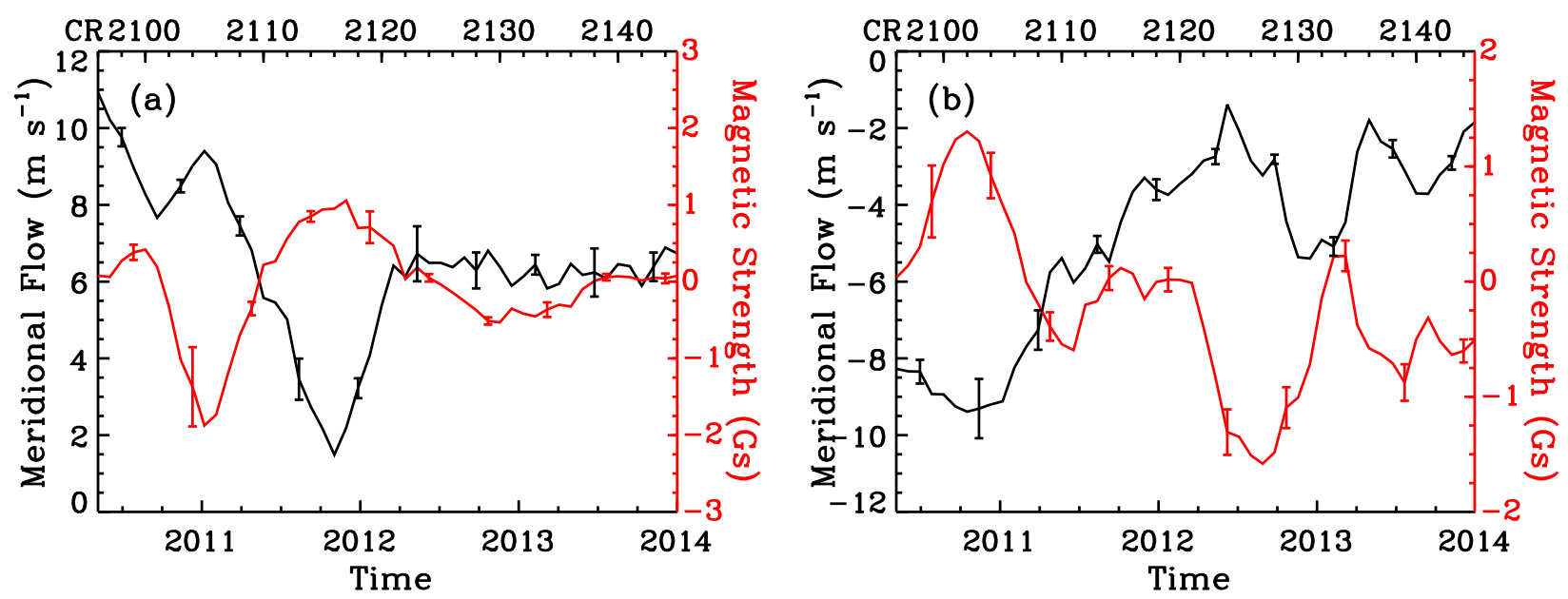

Fig. 5.- (a) Black curves are meridional-flow velocity at the depth of $0-1 \mathrm{Mm}$, as a function of time, averaged from the latitude $35^{\circ}-40^{\circ} \mathrm{N}$. Red curves are magnetic field from the same latitudinal bands. (b) Same as in panel (a) but for the latitude $40^{\circ}-45^{\circ} \mathrm{S}$. Note that the meridional flow speed increases with the vertical axis in panel (a) but decreases in panel (b), and the speed is displayed without the mean flow subtracted. 